\title{
Endocervical Cavity Anomaly Mimicking the Uterine Cavity and Delaying Diagnosis of Endometrial Adenocarcinoma: A Case Report
}

\author{
Meng-Yu Wu ${ }^{1,2} \mathbb{1}$, Dah-Ching Ding ${ }^{3,4}$, Tang-Yuan Chu ${ }^{3,4}$ and Mun-Kun Hong ${ }^{3,4, *}$ \\ 1 Department of Emergency Medicine, Taipei Tzu Chi Hospital, Buddhist Tzu Chi Medical Foundation, \\ New Taipei 231, Taiwan; skyshangrila@gmail.com \\ 2 Department of Emergency Medicine, School of Medicine, Tzu Chi University, Hualien 970, Taiwan \\ 3 Department of Obstetrics and Gynecology, Hualien Tzu Chi Hospital, Hualien 970, Taiwan; \\ dah1003@yahoo.com.tw (D.-C.D.); hidrchu@gmail.com (T.-Y.C.) \\ 4 Institute of Medical Science, Tzu Chi University, Hualien 970, Taiwan \\ * Correspondence: jeff06038@gmail.com; Tel.: +886-3-856-1825 (ext. 2169)
}

Received: 13 March 2018; Accepted: 23 March 2018; Published: 26 March 2018

\begin{abstract}
We report a rare case of endometrial cancer in an endocervical anomaly with a dilated and isolated endocervical cavity mimicking the uterine cavity. A 75-year-old woman suffered from persistent brownish watery vaginal discharge. The cytology of the fluid showed some atypical cells, and trans-vaginal ultrasonography disclosed two distinct compartments in the pelvis. Under the impression of hematometra and adnexal cystic tumor with solid part, dilation and curettage (D\&C) was done, but the pathology showed no evidence of malignancy. Although the hematometra and adnexal cyst disappeared afterward, the patient returned to the clinic with a complaint of persistent vaginal fluid two months later, and trans-rectal ultrasound revealed a cystic tumor with solid part again. Finally, endometrioid adenocarcinoma of endometrium was diagnosed by diagnostic laparoscopy and hysterectomy. This work highlights our experience in the diagnostic process and calls attention to the possibility of an occult endometrial cancer when evaluating postmenopausal women with continuous abnormal watery vaginal fluid and/or atypical cells shown in vaginal fluid cytology.
\end{abstract}

Keywords: endometrial cancer; atypical cell; acquired uterine abnormality; trans-rectal ultrasound; trans-vaginal ultrasound

\section{Introduction}

Endometrial cancer, which has an incidence of 12.9 per 100,000 women, is the most common gynecologic malignancy in developed countries, and the incidence has risen in recent years [1]. This cancer is often diagnosed at the early stage because of early symptoms of abnormal uterine bleeding. In general, the five-year survival rate of endometrial cancer is $70-80 \%$ for early stage and $20-60 \%$ for advanced stage [2,3]. Trans-vaginal ultrasound (TVUS) is an effective method of assessing endometrial lesions [4]. The sensitivity and the specificity of TVUS for detecting endometrial carcinoma in menopausal women are $96 \%$ and $61 \%$, respectively [5]. However, in rare cases with an abnormal uterus or cervix, such as arcuate or septate uterus or a dilated endocervical cavity as the reported case, TVUS alone is inadequate for diagnosing endometrial lesion.

This case report describes a uterine abnormality with a dilated and isolated endocervical cavity that mimicked the uterine cavity and initially confused the gynecologist. The purpose of this report is to highlight the pitfalls and to share our experience in diagnosing endometrial cancer in a case of endocervical anomaly. The association between atypical cells on vaginal cytology and endometrial 
cancer is also discussed. Hopefully, this work will help gynecologists avoid a potential misdiagnosis when evaluating postmenopausal women with continuous abnormal watery vaginal fluid and/or atypical cells shown in vaginal fluid cytology, which may indicate occult endometrial cancer.

\section{Case Presentation Section}

A 75-year-old nulligravida presented with underlying hypertension with well-controlled diabetes mellitus. She had visited our clinic for postmenopausal abnormal vaginal discharge five months earlier. Pelvic examination showed some watery brownish fluid with malodor in the vagina without visible cervical tumor. The cytology of the fluid showed some atypical cells (Figure 1A). Trans-vaginal ultrasonography disclosed two distinct abnormal compartments in the pelvis: one near the probe resembled a homogeneous hypoechogenic hematometra (Figure 1B), and another one near the left adnexa was a $41.3 \times 59.4-\mathrm{mm}$ cystic tumor with a solid component $(23.8 \times 24.3 \mathrm{~mm})$ (Figure 1C).
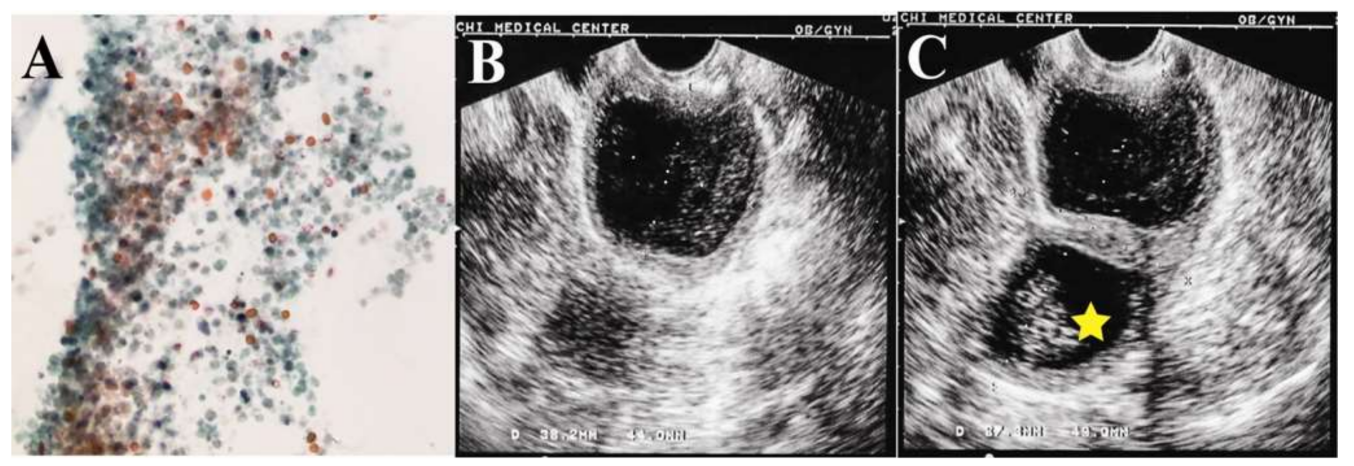

Figure 1. (A) The cytology of the fluid showed some atypical cells, granular debris of cell and lysed blood cells; (B) hypo-echoic hematoma in the pelvis; (C) cystic tumor with a $23.8 \times 24.3$-mm solid tumor (star) in the pelvis.

The abdominal computer tomography (CT) scan revealed an anteverted uterus with two distinct compartments: a thick-walled cystic tumor with solid lesion deviated to the left side pelvis (Figure 2A), and a lower lesion looked like fluid accumulation in the uterine cavity (Figure 2B). Laboratory examinations revealed that tumor markers were within normal ranges (Carcinoembryonic Antigen $<0.5 \mathrm{ng} / \mathrm{mL}$, Cancer Antigen 125: $13.7 \mathrm{IU} / \mathrm{mL}$, Cancer Antigen 19-9: $17.63 \mathrm{IU} / \mathrm{mL}$ ), and the fasting glucose level was $128 \mathrm{mg} / \mathrm{dL}$. Under the impression of endometrial and/or ovarian cancer, hysteroscopy and dilation and curettage (D\&C) were performed. Hysteroscopy showed dense old blood in a compact cavity, and a chronic inflammatory wall was noted after washing with normal saline.

One week after D\&C, the TVUS showed minimal retained fluid in the drained cavity (Figure 2C), and the adnexal cyst was not found. The pathology showed only hematoma with a few superficial endometrial glands and endocervical cells. Since there was no evidence of malignancy, and the patient was advised to return for follow up. However, the abnormal vaginal fluid persisted for two months afterward. She visited a gynecology oncologist in our hospital for a second opinion. TVUS showed similar findings to the previous follow up, but trans-rectal ultrasound (TRUS) revealed a soaked adnexa or endometrial tumor $(23.3 \times 9.6 \times 16.9 \mathrm{~mm})$ with a solid part (Figure 2D). 


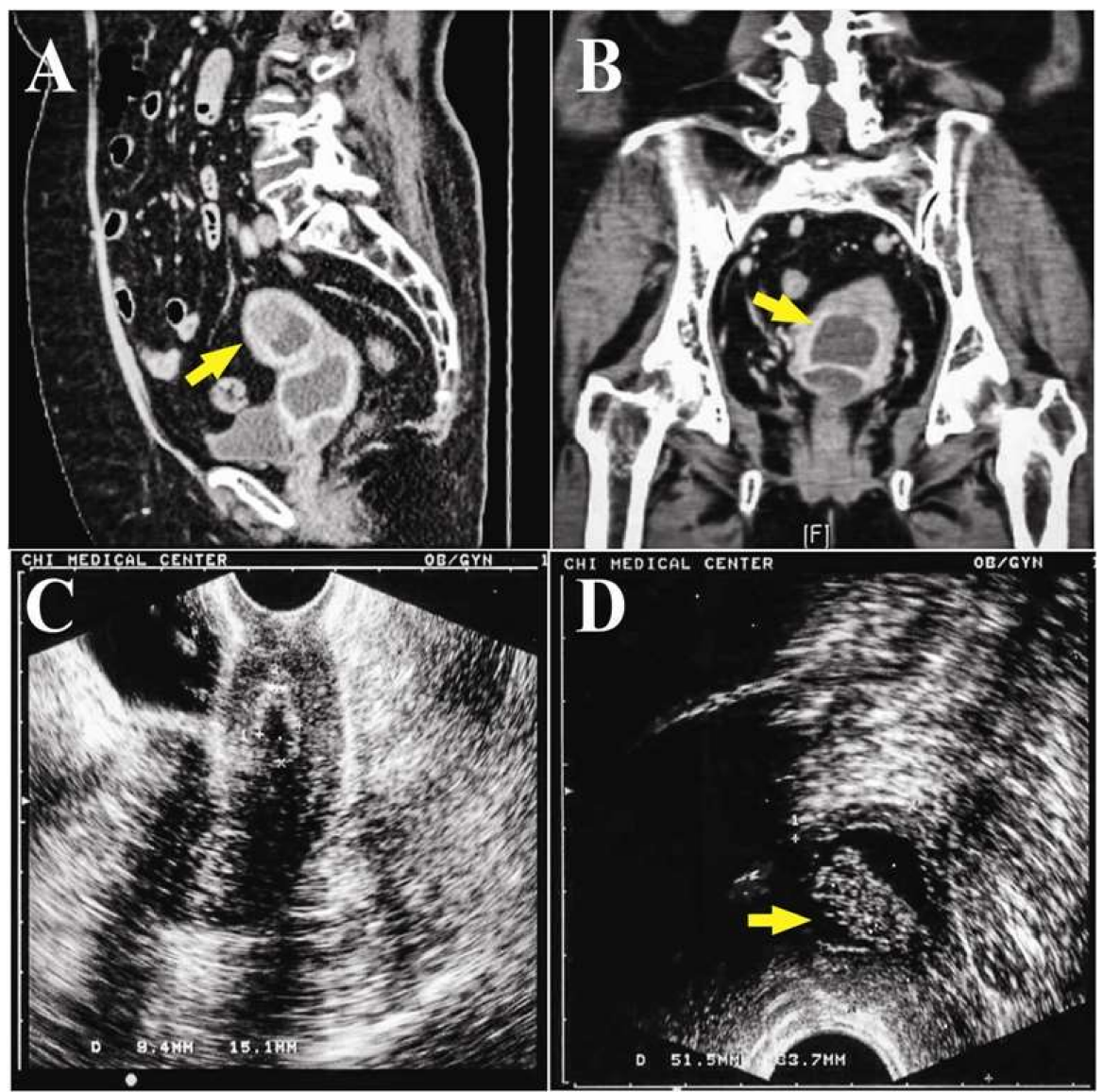

Figure 2. (A) The sagittal reformat CT scan revealed two distinct compartments: a thick-walled cyst (arrow) with a soft tissue lesion, and another one was a homogeneous fluid density cystic tumor in the pelvis; (B) two fluid-density compartments (arrow) in the pelvis in the coronal plane of the CT scan; (C) trans-vaginal ultrasound showed a confined and deflated cavity in the pelvis after dilation and curettage (D\&C); (D) trans-rectal ultrasound showed a cystic tumor with a $23.3 \times 9.6 \times 16.9$-mm solid part (arrow).

Hysteroscopy and D\&C were performed again, but only revealed a small and atrophic "uterine cavity". The pathological report of the D\&C was normal endometrial glands and chronic cervicitis. Under the impression of adnexal tumor with suspicion of fallopian tube or ovarian cancer, she received a diagnostic laparoscopy. During surgery, atrophic uterus, ovaries and fallopian tubes were noted, but no adnexal tumor was found (Figure 3A,B). Laparoscopic-assisted vaginal hysterectomy and bilateral salpingo-oophorectomy were done. When the uterus was opened, two cavities were noted: the true uterine cavity was filled with old blood and necrotic tumors, and the lower cavity was actually a dilated endocervical cavity. A 1.5-cm pseudo-polypoid tumor was found on the superficial endometrium in the true uterine cavity. Finally, a pathology report revealed endometrioid adenocarcinoma of endometrium, stage 1a, grade 3, with squamous differentiation (Figure 3C,D). After surgery, the symptoms were relieved, and the patient refused to receive complete staging surgery. The other adjuvant treatments, such as radiation therapy and brachytherapy, were suggested to this intermediate-risk patient due to grade 3 with squamous differentiation. However, the patient decided to observation after surgical intervention. During two years follow up, there was no evidence of local recurrence, and the patient received regular follow up at outpatient department. A written informed consent was obtained from the patient. 

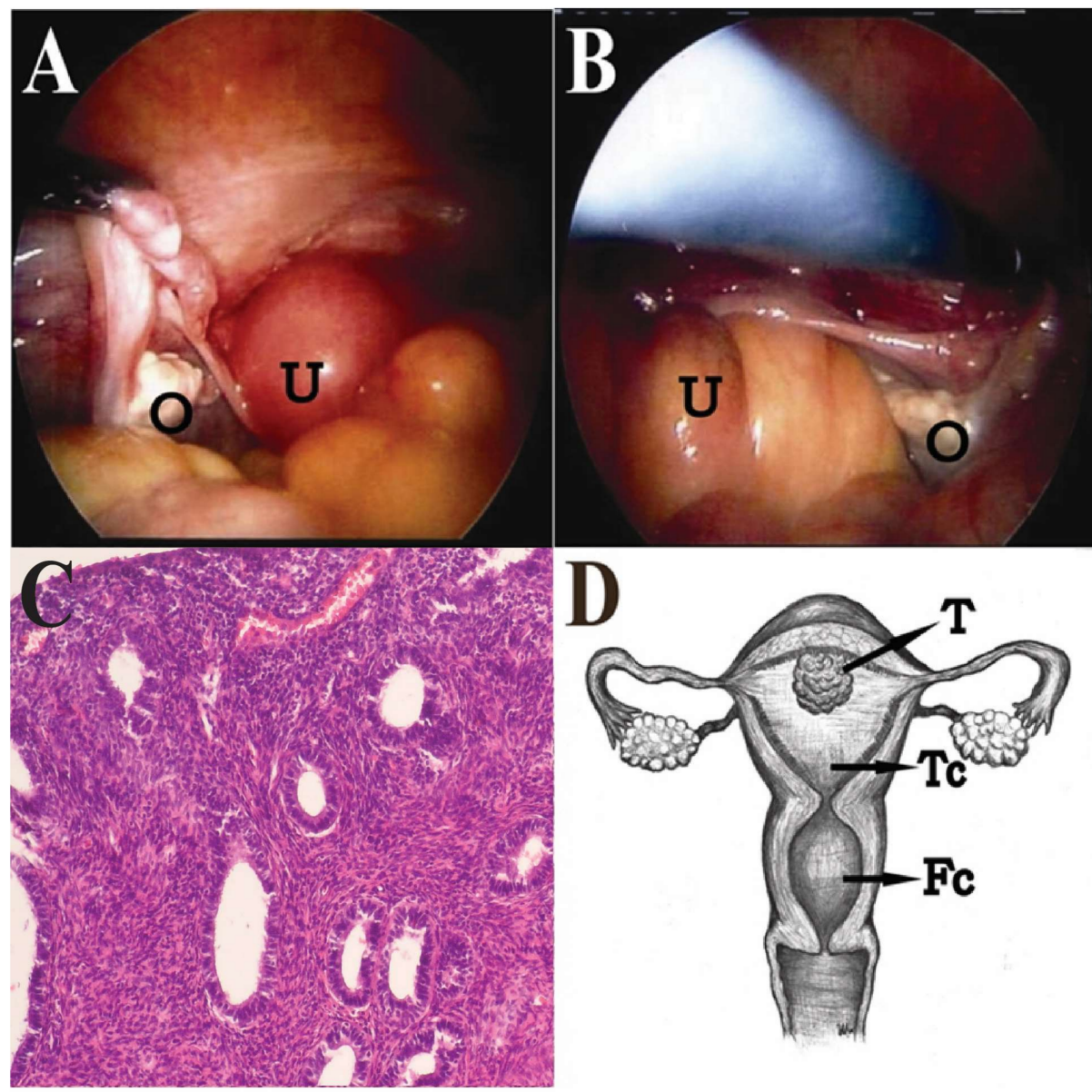

Figure 3. (A,B) The uterus (U) was small, and the fundus was smooth. The bilateral ovaries (O) and fallopian tubes were atrophic; (C) The pathology revealed endometrioid adenocarcinoma of endometrium with squamous differentiation (magnification, $\times 40$ ); (D) The gross view of anteverted uterus with left deviation showing two cavities: the true uterine cavity (Tc) was filled with old blood and a 1.5-cm pseudo-polypoid tumor $(\mathrm{T})$; the lower false cavity $(\mathrm{Fc})$ was a dilated endocervical cavity.

\section{Discussion}

Most uterine anomalies are congenital malformations resulting from abnormal development of the Müllerian ducts resulting in either failed fusion or septal resorption [6]. This report describes a rare case of dilated and isolated endocervical abnormality mimicking the uterine cavity and confusing the gynecologist on interpretation of the ultrasonography image. During the two times of hysteroscopy examination, the small and atrophic cavity was a dilated isolated endocervical cavity. Therefore, the gynecologist was attracted by this obvious finding and misunderstood it as the uterine cavity in this anteverted and left rotated uterus initially; in fact, the true uterine cavity had a pseudo-polypoid lesion that was also misunderstood as an adnexal cystic tumor. Similarly, on follow up after D\&C, the gynecologist was attracted by the obvious changed image of the drained "uterine cavity" and forget to check the pelvis anteriorly and posteriorly at a larger angle with the probe. In this patient, she had no previous ultrasound report. The endocervical abnormality cannot be defined as congenital or acquired malformation. We believe that it may be acquired malformation. We hypothesize that somehow the internal and external orifice of the cervix were initially so narrow that the fluid produced by the endometrial cancer could not efficiently be drained into the endocervix; similarly, the fluid produced by the endocervical gland could not efficiently be drained into the vagina. The uterine and endocervical cavities then dilated and formed two distinct cavities. During TVUS, the dilated endocervical cavity near the probe appeared to be a hematometra, while the true hematometra in this 
anteverted and left rotated uterus looked like an adnexal cystic tumor. Because of the difficulty in distinguishing the two cavities the first time and the pathology report of D\&C revealed no evident of malignancy, the gynecologist was hesitant to perform a further invasive procedure.

Although TVUS is an effective method for endometrial lesion assessment, TVUS alone is inadequate for this reported case. Few studies have reported the use of TVUS for detecting endometrial cancer in abnormal uterus. The TRUS is a commonly-used tool for evaluating prostatic carcinomas and is also used by some gynecology oncologists for evaluating local extension of cervical carcinomas [7]. In the literature, only one case of endometrial carcinoma with a small and markedly retroverted uterus detected by TRUS has been reported [8]. Gynecologists should consider using TRUS as an alternative tool when abnormal uterus is suspected. Indeed, in this case, the TRUS was important for clarifying the previous abnormal ultrasonography finding (adnexal cystic tumor) because it could not be repeated by TVUS on follow up. In addition, three-dimensional sonohysterography may be an alternative tool for a better assessment of the uterine cavity.

The CT scan and magnetic resonance imaging (MRI) study are important for assessing endometrial cancer, including staging, treatment planning and follow-up. The MRI is superior to CT and TVUS in evaluating endometrial cancer tumor size, stage and myometrial infiltration due to its excellent contrast-resolution in the soft tissue. The CT is a good alternative tool for patients who are contraindicated for MRI and is typically used to survey extrauterine disease. In this case, CT was used to confirm the trans-vaginal sonography finding. Although MRI is outstanding for soft components and provides more information for physicians, it was not done in our case because in clinical practice, the CT image is more rapid and has similar sensitivity and specificity as MRI. However, the hysteroscopic and D\&C findings did not support the existence of malignancy. Fortunately, the cytology of the vaginal fluid revealed atypical cells, and persistent symptoms of watery vaginal discharge alerted the gynecology oncologist to perform diagnostic laparoscopy at the other time of investigation.

Due to profound abnormal fluid found in vagina, it was sent for cytology at the first time of pelvic examination. Atypical cells were reported, this is an important hint for pre-malignant disease or malignancies from genital tract. Atypical epithelial cells are the presence of nuclear enlargement and pleomorphism and mainly can be classified as squamous or glandular [9]. Atypical squamous cells (ASC) are usually from cervix or vagina and account for $2.8 \%$ of cervical cytology samples [10]. While atypical glandular cells (AGC), usually from the endocervix or endometrium, account for $0.05-2.1 \%$ of cervical cytology samples [11], of them, approximately 35\% are associated with premalignancy or malignancy [12-14]. Atypical cells are a well-established risk factor for endometrial carcinoma. The endometrial cytology with atypical cells has about a 25 percent likelihood of progressing to carcinoma [15]. The detection of atypical cells in vaginal fluid cytology was a crucial clue supporting the gynecology oncologist for further investigation. This case report is an example of how uterine anomalies can cause gynecologists to misinterpret TVUS and delay diagnosis of a malignant disease.

Acknowledgments: This work was not supported by any fund.

Author Contributions: Meng-Yu Wu wrote the paper and contributed to the organization of the figure. Dah-Ching Ding and Mun-Kun Hong provided conceptual input. Tang-Yuan Chu and Mun-Kun Hong proofread and organized the manuscript. All authors reviewed the final version of the manuscript.

Conflicts of Interest: The authors declare no conflict of interest.

\section{References}

1. Jemal, A.; Bray, F.; Center, M.M.; Ferlay, J.; Ward, E.; Forman, D. Global cancer statistics. CA Cancer J. Clin. 2011, 61, 69-90. [CrossRef] [PubMed]

2. Creasman, W.T.; Odicino, F.; Maisonneuve, P.; Quinn, M.A.; Beller, U.; Benedet, J.L.; Heintz, A.P.; Ngan, H.Y.; Pecorelli, S. Carcinoma of the corpus uteri. FIGO 26th Annual Report on the Results of Treatment in Gynecological Cancer. Int. J. Gynaecol. Obstet. 2006, 95, S105-S143. [CrossRef]

3. Lewin, S.N.; Wright, J.D. Comparative Performance of the 2009 International Federation of Gynecology and Obstetrics' Staging System for Uterine Corpus Cancer. Obstet. Gynecol. 2011, 117, 1226. [CrossRef] [PubMed] 
4. Hosoi, A.; Ueda, Y.; Shindo, M.; Nakagawa, S.; Matsuzaki, S.; Kobayashi, E.; Kimura, T.; Yoshino, K.; Fujita, M.; Kimura, T. Endometrial thickness measured by ultrasonography in postmenopausal patients with endometrial carcinoma has significance, irrespective of histological subtype. Int. J. Gynecol. 2013, 23, 1266-1269. [CrossRef] [PubMed]

5. Smith-Bindman, R.; Kerlikowske, K.; Feldstein, V.A.; Subak, L.; Scheidler, J.; Segal, M.; Brand, R.; Grady, D. Endovaginal ultrasound to exclude endometrial cancer and other endometrial abnormalities. JAMA 1998, 280, 1510-1517. [CrossRef] [PubMed]

6. Bermejo, C.; Martinez Ten, P.; Cantarero, R.; Diaz, D.; Perez Pedregosa, J.; Barron, E.; Labrador, E.; Ruiz Lopez, L. Three-dimensional ultrasound in the diagnosis of Mullerian duct anomalies and concordance with magnetic resonance imaging. Ultrasound Obstet. Gynecol. 2010, 35, 593-601. [CrossRef] [PubMed]

7. Zaritzky, D.; Blake, D.; Willard, J.; Resnick, M. Transrectal ultrasonography in the evaluation of cervical carcinoma. Obstet. Gynecol. 1979, 53, 105-108. [PubMed]

8. Holland, C.M.; Berman, L.H.; Crawford, R.A. A case of endometrial carcinoma detected using transrectal ultrasound. Int. J. Gynecol. Cancer 2004, 14, 551-552. [CrossRef] [PubMed]

9. Lacey, J.V., Jr.; Chia, V.M. Endometrial hyperplasia and the risk of progression to carcinoma. Maturitas 2009, 63, 39-44. [CrossRef] [PubMed]

10. Katki, H.A.; Schiffman, M.; Castle, P.E.; Fetterman, B.; Poitras, N.E.; Lorey, T.; Cheung, L.C.; Raine-Bennett, T.; Gage, J.C.; Kinney, W.K. Benchmarking CIN 3+ risk as the basis for incorporating HPV and Pap cotesting into cervical screening and management guidelines. J. Lower Genit. Tract Dis. 2013, 17, S28-S35. [CrossRef] [PubMed]

11. Marques, J.P.; Costa, L.B.; Pinto, A.P.; Lima, A.F.; Duarte, M.E.; Barbosa, A.P.; Medeiros, P.L. Atypical glandular cells and cervical cancer: Systematic review. Rev. Assoc. Med. Bras. 2011, 57, 234-238. [CrossRef] [PubMed]

12. DeSimone, C.P.; Day, M.E.; Tovar, M.M.; Dietrich, C.S., 3rd; Eastham, M.L.; Modesitt, S.C. Rate of pathology from atypical glandular cell Pap tests classified by the Bethesda 2001 nomenclature. Obstet. Gynecol. 2006, 107, 1285-1291. [CrossRef] [PubMed]

13. Sharpless, K.E.; Schnatz, P.F.; Mandavilli, S.; Greene, J.F.; Sorosky, J.I. Dysplasia associated with atypical glandular cells on cervical cytology. Obstet. Gynecol. 2005, 105, 494-500. [CrossRef] [PubMed]

14. Veljovich, D.S.; Stoler, M.H.; Andersen, W.A.; Covell, J.L.; Rice, L.W. Atypical glandular cells of undetermined significance: A five-year retrospective histopathologic study. Am. J. Obstet. Gynecol. 1998, 179, 382-390. [CrossRef]

15. Canavan, T.P.; Doshi, N.R. Endometrial cancer. Am. Fam. Phys. 1999, 59, 3069-3077. 